\title{
Sprichwörter im Wörterbuch
}

\author{
Vida Jesenšek (Maribor)
}

\begin{abstract}
This text aims to contribute to the discussion of the lexicographical treatment of proverbs. Discussed are some selected problem areas that, in terms of proverbs, become evident in planning and preparing dictionaries and comparable linguistic resources. Considerations are based on the partial results of the EU-funded research project SprichWort ${ }^{1}$. In this project, a multilingual proverb database is created and developed.
\end{abstract}

\section{$1 \quad$ Einleitung}

Dieser Text hat das Ziel, zur Diskussion über die lexikographische Behandlung von Sprichwörtern beizutragen. Diskutiert werden einige ausgewählte Problembereiche, die sich hinsichtlich der Sprichwörter bei der Konzipierung und Erarbeitung von Wörterbüchern und vergleichbaren Sprachressourcen zeigen. Die Überlegungen basieren auf den Teilergebnissen des EU-geförderten Forschungsprojekts SprichWort ${ }^{2}$, in dem unter anderem eine mehrsprachige Sprichwort-Datenbank entwickelt und erarbeitet wird.

\section{Sprichwörter in der gängigen lexikographischen Praxis - Stand der Dinge}

Sprichwörter waren bis zu den 80er Jahren des 20. Jahrhunderts relativ selten Gegenstand der metalexikographischen Forschung. Dies mag erstaunen, da sie in den allgemeinen Wörterbüchern doch in der Regel verzeichnet sind und spezielle parömiologische Sammlungen in vielen Sprachen eine lange Tradition sowie Beliebtheit bei den Lesern nachweisen können. Einerseits liegen Gründe dafür in der Tatsache, dass die Zusammenstellung von Sprichwort-Sammlungen seit ihren Anfängen prinzipiell die Domäne der ethnographischen und ethnologischen Forschung gewesen ist. Andererseits sind Sprichwörter in vielen allgemeinen Lexika nur als Belegmaterial zu lemmatisierten EinwortLexemen, die als Sprichwort-Komponenten auftreten, verzeichnet, während eine reflektierte Behandlung als relativ autonome Lexikon-Einheiten in der Regel fehlt.

Im neuesten bibliographischen Werk Wolfgang Mieders, des unermüdlichen SprichwortForschers (International Bibliography of Paremiology and Phraseology 2009) sind in 2 Bänden rund 10000 bibliographische Angaben zu parömiologisch (wohl aber auch generell phraseologisch) orientierten Publikationen verzeichnet, die weltweit in den letzten zwei Jahrhunderten erschienen sind. Darunter findet man zwar etliche Titel zur sprichwortbezogenen Thematik, jedoch hat man den Eindruck, dass die aktuelle Metalexikographie Sprichwörter eher ausnahmsweise als Forschungsobjekt fokussiert. ${ }^{3}$

So verwundert es nicht, wenn die gängige lexikographische Praxis hinsichtlich der Sprichwörter ähnliche Mängel aufweist, wie sie mehrere Male schon für die Phraseographie

\footnotetext{
1 Available at http://www.sprichwort-plattform.org.

2 Verfügbar unter http://www.sprichwort-plattform.org (Stand: 26. September 2010).

3 Eine konzise Übersicht über ausgewählte theoretische Sprichwort-Publikationen gibt Kispál (2007).
} 
generell festgestellt worden sind (vgl. Burger 2010, Dobrovol'skij 2009, Ďurčo 2005, Grzybek 1992, Hallsteinsdóttir 2006 und 2009, Jesenšek 2006 und 2008, Kispál 2000, Mieder 1984 und 1989). Problemstellen, die gleicherweise in der allgemeinen und speziellen ein- und zweibzw. mehrsprachigen Lexikographie aktuell sind, betreffen sowohl die Makro- als auch die Mikrostruktur eines Wörterbuchs.

\subsection{Makrostrukturelle Fragen}

Makrostrukturelle Problembereiche betreffen die Sprichwort-Definition, die Selektionsmethoden und die Einordnung der Sprichwörter in die Makrostruktur eines Wörterbuchs.

Das Grundproblem liegt wohl darin, dass zwischen den unterschiedlichen phraseologischen Lexikon-Einheiten in der Regel nicht klar unterschieden wird und dass Sprichwörter dementsprechend definitorisch überhaupt nicht oder nur vage festgelegt sind. Vor allem betrifft dies eine unzureichende oder sogar völlig fehlende Unterscheidung zwischen satzund nichtsatzwertiger Phraseologie, vgl. dazu Betrachtungen zur deutschen und englischen allgemeinen Lexikographie und Parömiologie bei Mieder (2003) und Kispál (2007). Ähnliches muss Jesenšek (2007) zum Status der Sprichwörter in den allgemeinen Wörterbüchern für das Slowenische feststellen. Anhand von DUW (2001) lässt sich die definitorische Problematik ebenso leicht illustrieren:

Laut Erläuterung der verwendeten Abkürzungen werden Sprichwörter mit Spr markiert, mir R dagegen Redensarten (DUW 2001: 26); Sprichwort ist "kurzer, einprägsamer Satz, der eine praktische Lebensweisheit enthält" (ebd.: 1493), Redensart wird als "formelhafte Verbindung von Wörtern, die meist als selbstständiger Satz gebraucht wird" definiert (ebd.: 1286). Unter dem Lemma Ende finden wir dann Folgendes: "... R (scherzh.) alles hat ein E., nur die Wurst hat zwei; bis zum bitteren E.; Spr E. gut, alles gut..." (ebd.: 460f.); unter dem Lemma Not etwa: "...Spr Wenn die N. am größten, ist Gottes Hilfe am nächsten /.../ R N. macht erfinderisch..." (ebd.: 1145).

Weiterhin ist die Auswahl der in ein Wörterbuch aufgenommenen Sprichwörter in vielen Fällen methodologisch nicht nachvollziehbar, zumal die Selektionskriterien und -prinzipien in der Regel nicht bekannt gegeben werden. Lexikographische Umtexte in etlichen Wörterbüchern bringen in dieser Hinsicht eher vage Aussagen hervor. Oft kann man annehmen, dass die Aufnahme oder Nichtaufnahme von Sprichwörtern einfach von ihrer Präsenz in den bereits existierenden Wörterbüchern und sonstigen parömiologischen Sammlungen abhängt und/oder sie beruht lediglich auf der Sprachkompetenz des Lexikographen und seinen intuitiven Entscheidungen, was allerdings die Qualität eines lexikographischen Nachschlagewerks ernsthaft beeinträchtigt. Folglich erscheint auch die makrostrukturelle Einordnung der polylexikalen Sprichwörter in vielen Wörterbüchern weniger systematisch und konsequent, was eine optimale Auffindbarkeit der Sprichwörter im Wörterbuch beinahe ausschließt (vgl. Kispál 2007).

Makrostrukturell sind also folgende Problemfelder bei der lexikographischen Behandlung von Sprichwörtern identifizierbar:

- Wie definiert man das Sprichwort als eine Art der satzwertigen Phraseme?

- Wie legt man den Status von Sprichwörtern für ein zu erstellendes Wörterbuch fest? Werden sie als eigenständige komplexe Lexeme behandelt, dementsprechend lemmatisiert und somit zu Elementen der Makrostruktur erklärt? Haben sie lediglich die Beispielfunktion zu einzelnen Lexemen, die als Sprichwort-Komponenten vorkommen? Ist beides zugleich der Fall? 
- Nach welchen Prinzipien und Kriterien werden Sprichwörter für ein zu erstellendes Wörterbuch selektiert?

- Nach welchen Prinzipien werden sie - falls sie als Lemmata vorkommen - in die Makrostruktur eines Wörterbuchs integriert?

- Wie erreicht man ihre optimale Auffindbarkeit im Wörterbuch?

\subsection{Mikrostrukturelle Fragen}

Noch komplexer scheinen die mikrostrukturellen Aspekte der lexikographischen SprichwortBehandlung zu sein. Kispál (2007) thematisiert hierzu drei Problembereiche (Lemmatisierung, Varianz und Angaben), muss aber euphemistisch feststellen, dass wegen vieler ungeklärter mikrostruktureller Aspekte die Wörterbuchartikel in SprichwortWörterbüchern extrem heterogen sind: "In Sprichwörterbüchern ist die Vielfalt der Wörterbuchartikel noch auffallender als in allgemeinen Wörterbüchern" (Kispál 2007: 418).

Sieht man von einem bloßen Abschreiben aus den bestehenden Wörterbüchern und Sammlungen ab, so sind im Hinblick auf die gegenwärtige lexikographische Praxis und den Stand der phraseologisch-phraseographischen Forschung für eine adäquate lexikographische Bearbeitung von Sprichwörtern folgende Fragen relevant:

- Wie lässt sich die lexikographische Ausgangsform eines Sprichworts (die so genannte Nennform) aus den textuellen Realisierungen, die auch stark variabel sein können, herausfiltern? Welche Methoden sind hierbei anzuwenden? Wie kommt man zu einem zuverlässigen, empirisch gesicherten Resultat, welches den lexikalisierten Usus repräsentiert und somit als lexikographisch relevant gelten kann?

- Wie soll die lexikographische Darstellung der Variabilität von Sprichwort-Strukturen erfolgen? Welche sind Kriterien, nach denen usuelle lexikalische, morphologische, syntaktische, orthographische Sprichwort-Varianten ermittelt und festgelegt werden können? Wie differenziert man textuelle und/oder autorspezifische Modifikationen?

- Wie lässt sich die in der Regel komplexe inhaltliche Struktur von Sprichwörtern lexikographisch adäquat erfassen? Wie soll eine benutzerorientierte Bedeutungsangabe ausformuliert werden?

- Wie kommt man zu den Daten über stilistische und pragmatische Eigenschaften der Sprichwörter und wie präsentiert man sie?

- Wie weist man auf die syntaktische Einbettung der Sprichwörter im Text hin, zumal bekannt ist, dass sie nicht ausschließlich als Zitatsätze verwendet werden? Wie, nach welchen Methoden ermittelt man repräsentative Sprachdaten dazu?

- Wie präsentiert man die anderssprachige Äquivalenz in zwei- und mehrsprachigen Wörterbüchern, um das gesamte Inhaltsspektrum des ausgangssprachlichen Sprichworts zu decken?

- Wie wählt man textuelle Belege zum Gebrauch von Sprichwörtern aus? Unter Berücksichtigung welcher Kriterien und Anwendung welcher Methoden werden diese aus den zur Verfügung stehenden Textkorpora und sonstigem Sprachmaterial ermittelt?

- Inwiefern und bei welchen Typen von Wörterbüchern sind etymologische und/oder kulturgeschichtliche Angaben zu einzelnen Sprichwörtern von Bedeutung? Wie sollen sie formuliert werden? 
- In welchen Typen von Sprichwort-Wörterbüchern sind Frequenzangaben zur Vorkommenshäufigkeit relevant? Nach welchen Methoden werden sie vermittelt, um als repräsentativ zu gelten?

Verfolgt man eine fachlich begründete lexikographische Praxis, so sollten bei der Konzeption und Erarbeitung von Wörterbüchern alle diese Aspekte berücksichtigt werden. Es gilt nämlich, dass "Varianten, Bedeutungen, Funktionen, Stilschichten usw. zu einem wissenschaftlich fundierten Lexikon gehören" (Mieder 1999: 23). Inwiefern sie dann in einem konkreten Wörterbuch realisiert werden, das hängt vom jeweiligen Wörterbuchtyp und von den vorgesehenen Wörterbuchfunktionen ab.

Die obige Diskussion der Problembereiche in der lexikographischen Behandlung von Sprichwörtern erfolgte primär anhand der metalexikographischen Überlegungen und lexikographischen Praxis in der Erstellung von klassischen Printwörterbüchern und ihren digitalisierten Formaten, sie ist aber genauso aktuell bei der Konzipierung und Erstellung von Sprachdatenbanken und ähnlichen elektronischen Sprachressourcen. Hierzu sind bisher mehrere Publikationen erschienen, die sich in erster Linie mit der korpusbasierten Erhebung von lexikographisch relevanten Sprachdaten beschäftigen (vgl. Steyer 2001, 2004, 2009, vgl. die Online-Bibliographie zur elektronischen Lexikographie OBELEX ${ }^{4}$ ). Meist beziehen sich die Texte auf das Lexikon an sich, einige thematisieren auch spezielle sprichwortbezogene Fragen, vor allem die Selektionsfrage (vgl. Baur/Chlosta 1996, Čermák 2003, Ďurčo 2004), wohl aber auch die Konzeption der lexikographischen Sprichwortbeschreibung (Ďurčo 2005), wo eine komplexe, mehrdimensionale, holistische Beschreibung angestrebt wird.

Auf der Basis derartiger Überlegungen wurden bisher mehrere Beschreibungskonzepte für Sprichwörter entwickelt, eines davon auch im Forschungsprojekt SprichWort. In seinen Wesenszügen wird dieses im Folgenden dargestellt und anschließend kurz diskutiert.

\section{Das Projekt SprichWort}

Das Hauptziel des Projekts SprichWort ${ }^{5}$ ist die Entwicklung und Erstellung einer online Lernplattform zur Vermittlung und zum Erlernen von ausgewählten Sprichwörtern in fünf Sprachen, die zueinander jeweils Nachbarsprachen sind: Deutsch, Slowenisch, Slowakisch, Tschechisch und Ungarisch. Die Auswahl der beteiligten Sprachen ist im Einklang mit den aktuellen sprachpolitischen Richtlinien der EU. Danach sollten lern- und lernerorientierte Vorhaben unterstützt werden, die zur Stärkung und Verbreitung von Kenntnissen der Nachbarsprachen sowie von weniger gesprochenen und gelernten europäischen Sprachen beitragen und zugleich die Sensibilisierung für diese Sprachen fördern. Die Plattform ssieht drei konstitutive und miteinander verlinkte Hauptkomponenten vor: eine fünfsprachige Sprichwort-Datenbank, interaktive und multimedial aufbereitete didaktische Inhalte und eine Nutzer-Community. Im Weiteren bezieht sich der Text nur auf die Datenbank-Komponente der Plattform.

Makrostrukturell entsteht die Datenbank ausgehend von der deutschen Sprache, die im Projekt den Status der Ausgangsprache hat. Korpusmethodisch wird ein Inventar von aktuell üblichen und in thematischer Hinsicht didaktisch relevanten Sprichwörtern (300 Einträge)

\footnotetext{
4 Verfügbar unter http://hypermedia.ids-mannheim.de/pls/lexpublic/bib.ansicht (Stand: 28. Dezember 2010).

5 Das Projekt SprichWort (143376-2008-LLP-SI-KA2-KA2MP, 2008-2010) wird von der Europäischen Kommission im Rahmen der Aktion Lebenslanges Lernen gefördert. Das Projekt koordiniert die Philosophische Fakultät der Universität Maribor (Slowenien) mit ihrem Germanistischen Institut. Weitere Projekt-Partner sind Germanisten, Slawisten, Hungaristen und Lernsoftware-Entwickler von der Technischen Universität Graz (Österreich), vom Institut für deutsche Sprache Mannheim (Deutschland), von der Universität der Hlg. Cyril und Methodius Trnava (Slowakei), der Tomas-Bata-Universität Zlin (Tschechien) und der Universität Szeged (Ungarn).
} 
erarbeitet. Im Projekt werden Sprichwörter als propositionale Phraseme mit unikalem Charakter aufgefasst, die also semantisch und syntaktisch den phraseologischen Kriterien der Idiomatizität und Stabilität entsprechen, meist eine metaphorische Natur haben und auf tradierte menschliche und kulturelle Erfahrungen hinweisen. Sie werden als "in sich geschlossene Sätze, die durch kein lexikalisches Element an den Kontext angeschlossen werden müssen" verstanden, die also "als selbstständige 'Mikrotexte' aufgefasst werden können" (Burger 2010: 106). Didaktische Relevanz gründet hauptsächlich auf zwei Faktoren: zum einen auf einem hohen Grad der Aktualität im gegenwärtigen Sprachgebrauch (korpusermittelte statistische Häufigkeit) und zum anderen auf der inhaltlichen Relevanz gemäß dem Gemeinsamen europäischen Referenzrahmen für Sprachen (Profile Deutsch als Umsetzung des Referenzrahmens für Deutsch). Neben der strikten korpusbasierten Erhebung parömiologischer Sprachdaten ist ein bedeutendes Merkmal dieser Datenbank die Verlinkung mit externen Sprachressourcen. Parallel mit der Datenbankerstellung entsteht somit ein methodisches Vorgehensmodell für künftige Selektionen und kontrastiv-lexikographische Beschreibungen von Sprichwörtern (vgl. Jesenšek 2010).

Geht man von den aktuellen metalexikographischen Positionen aus, wonach der Benutzer eines lexikographischen Nachschlagewerks (wozu auch viele Sprachdatenbanken gezählt werden können), seine Konzeption und Erarbeitung vorgibt, so lassen sich hauptsächlich zwei Gruppen der anvisierten Benutzer der SprichWort-Datenbank festlegen, die für die vorliegenden Überlegungen besonders von Interesse sind: (1) Lerner der beteiligten Sprachen als Fremdsprachen und (2) Lehrer der beteiligten Sprachen als Fremdsprachen. ${ }^{6}$ Für beide Benutzergruppen ist anzunehmen, dass sie Sprachdatenbanken in fremdsprachlichen Lernprozessen benutzen. Als ein zusätzlicher Anwendungsbereich bieten sich Sprachmittlungstätigkeiten an, die entweder in der Fremdsprache (Schreiben, Lesen) oder zwischen zwei Sprachen (Übersetzen, Dolmetschen) stattfinden.

Aufgrund einer relativ umfangreichen linguistischen Beschreibung einzelner Sprichwörter ist aber durchaus erwartbar, dass die SprichWort-Datenbank auch für Sprachmittlung in der Muttersprache relevant ist.

Situationen, in denen die genannten Benutzergruppen eine Sprachdatenbank konsultieren, sind somit folgende:

1. das gestörte Rezipieren in der Fremdsprache,

2. das gestörte Produzieren in der Fremdsprache,

3. das gestörte Übersetzen in die oder aus der Fremdsprache,

4. der Erwerb und/oder das Erlernen einer Fremdsprache,

5. die Konsultation bei der Unterrichtsvorbereitung. ${ }^{7}$

Es wird durch diese Situationen deutlich, dass sowohl die passive als auch die aktive Sprichwort-Kompetenz bei den Benutzern berücksichtigt wird. In Bezug darauf wurde im Projekt ein lexikographisches Beschreibungskonzept entwickelt, auf dessen Grundlage die Datenbank-Struktur festgelegt wurde. Aufgrund der Komplexität der Beschreibungsdimensionen sind auch Linguisten als eine weitere angestrebte Benutzergruppe der Datenbank angesprochen. Im Folgenden werden einzelne Aspekte der Sprichwortbeschreibung näher dargestellt.

6 Darüber hinaus dürfte die Datenbank auch für Fremdsprachendidaktiker, Experten im Bereich der ELernmaterialentwicklung, Fremdsprachenlehrerausbilder und nicht zuletzt für alle, die sich für Sprichwörter interessieren, von Nutzen sein.

7 Vgl. Überlegungen zum Benutzer und zur Benutzung von mehrsprachigen phraseologischen lexikographischen Nachschlagewerken in Jesenšek (2005). 


\subsection{Aspekte der lexikographischen Beschreibung von Sprichwörtern in der SprichWort-Datenbank}

\subsubsection{Sprichwort-Nennform}

Unter der Nennform wird die Kernform des Sprichworts mit Satzendezeichen angegeben. Hiermit verbunden ist die lexikographische Gegenstandsdefinition, die bekannterweise nicht wenige Probleme bereitet. Die Kernform eines jeden Sprichworts, die für alle beteiligten Sprachen strikt korpusanalytisch ermittelt wird, ist in der Regel die frequenteste Form, während andere relativ oft vorkommende Formen als Sprichwort-Varianten aufgefasst und dementsprechend angegeben werden:

Ausnahmen bestätigen die Regel. (Kernform, die meisten Treffer im deutschen referentiellen Textkorpus)

$\rightarrow$ Die Ausnahme bestätigt die Regel. (Sprichwort-Variante)

\subsection{2 Äquivalente in anderen Sprachen}

Hier werden Links zu den Sprichwort-Äquivalenten in anderen beteiligten Sprachen platziert. Wenn es sich nicht um eine 1:1-Entsprechung handelt, werden Kommentare mit der wortwörtlichen Übersetzung eingefügt:

Die Sonne bringt es an den Tag.

$\rightarrow$ slowenisch: "Nič ni tako skrito, da ne bi bilo/postalo očito."

$\rightarrow$ Kommentar zum slow. Sprichwort: Die wörtliche Übersetzung: Nichts ist so versteckt, dass es nicht sichtbar wäre/würde.

\subsubsection{Komponenten}

Die Komponentenangaben ermöglichen den Zugriff auf die Sprichwort-Artikel über einzelne Sprichwort-Komponenten. Es werden nur die autosemantischen Komponenten ausgezeichnet und zwar sowohl als Lemma wie auch als die Flexionsform, die im Sprichwort vorkommt.

\subsubsection{Bedeutung(en)}

Die Bedeutungserläuterungen werden in der Regel nicht aus den Wörterbüchern übernommen, sondern anhand der Korpusbelege oder anderer, vor allem textueller Quellen erarbeitet. Angestrebt wird eine standardisierte Beschreibungssprache, sodass alle Bedeutungsbeschreibungen mit Formeln wie "Sagt man, wenn jmd./etw. ... / Sagt man für eine Situation, in der ... / Sagt man dafür, dass ..." eingeleitet werden:

Der Ton macht die Musik.

$\rightarrow$ Sagt man dafür, dass es für das Erreichen eines Ziels nicht allein darauf ankommt, was man äußert, sondern vor allem auf welche Art und Weise man es tut.

Angaben zu pragmatischen Aspekten des Sprichwortinhaltes werden in der Regel unter der Rubrik Gebrauchsbesonderheiten abgehandelt. Hier gilt allerdings eine Ausnahme: Wenn pragmatische Aspekte für die Bedeutung des Sprichwortes konstitutiv sind, das heißt, wenn der Sinn des Sprichwortes nicht entsprechend semantisiert werden kann, ohne auf die Pragmatik einzugehen, dann fließt die pragmatische Angabe in die Bedeutungsbeschreibung ein:

Die dümmsten Bauern haben die dicksten Kartoffeln.

$\rightarrow$ Sagt man abwertend, wenn jemand ohne große Anstrengungen und unverdient Erfolg hat. 
In den Bedeutungsparaphrasen werden Schlüsselwörter (autosemantische Lexik, auch Wortverbindungen) gesondert ausgezeichnet und in einem Glossar abgelegt. So entsteht eine dynamische Taxonomie möglicher Themenwörter, über die man gezielt auf Sprichwörter zu bestimmten Themenkomplexen zugreifen kann:

Der Ton macht die Musik.

$\rightarrow$ Sagt man dafür, dass es für das Erreichen eines Ziels nicht allein darauf ankommt, was man äußert, sondern vor allem auf welche Art und Weise man es tut.

$\rightarrow$ Schlüsselbegriffe: [Art und Weise], [äußern], [Erreichen eines Ziels]

Die so dynamisch entstehende Liste kann auch als Formulierungshilfe benutzt werden, indem schon vorhandene Schlüsselwörter für die Bedeutungsbeschreibungen verwendet werden können.

\subsubsection{Gebrauchsbesonderheiten}

Mit Gebrauchsbesonderheiten sind jene konnotativen und pragmatischen Aspekte des sprichworteigenen Inhaltsplanes gemeint, die in den Korpusbelegen häufig zu beobachten sind, die aber nicht auf alle textuellen Verwendungen des Sprichworts verallgemeinerbar sind. Darunter verstanden werden z.B. Sprechakt, kommunikative Funktion, Kommunikationssituation, Konnotation (positiv, negativ, rangierend, auf-/abwertend), stilistische Markiertheit, Auffälligkeit des Vorkommens in einer Domäne oder Textsorte, Auffälligkeit des Vorkommens unter arealem Aspekt, Auffälligkeit in Hinblick auf Gruppenoder Sondersprache, Auffälligkeit hinsichtlich der Präsenz in der Gegenwartssprache u. Ä.:

Es ist noch kein Meister vom Himmel gefallen.

$\rightarrow$ In den Korpusbelegen wird mit dem Sprichwort häufig eine Beruhigung ausgedrückt: Es wird z. B. darauf verwiesen, dass jeder, auch der größte Könner, irgendwann einmal ein Anfänger war und man deswegen bei Schwierigkeiten nicht an den eigenen Fähigkeiten zweifeln oder aufgeben muss.

In bestimmten Korpusbelegen wird mit dem Sprichwort

- die Mahnung ausgedrückt, dass man nicht glauben solle, etwas ohne viel Übung und Ausdauer gleich gut zu können

- auf die Ausbildung von Handwerksmeistern oder den Mangel an guten Meistern Bezug genommen.

Die Beschreibungen von Gebrauchsbesonderheiten sind prinzipiell Abstrahierungen aus entsprechenden Korpusbelegen, auf die jeweils verwiesen wird.

\subsubsection{Varianten}

Das Konzept der Varianz basiert auf Usualität. Eine Variante ist eine typische, usuelle Veränderung der Sprichwortstruktur. Es werden nur Varianten dokumentiert, die eine gewisse Rekurrenz in den Korpusbelegen aufweisen (obligatorisches mehrfaches Vorkommen im Korpus), während okkasionelle, einmalige Abwandlungen (Modifikationen) nicht berücksichtigt werden.

Es wird zwischen Formvarianten und varianter Ersetzung von Komponenten unterschieden. Formvarianten meinen mehrere verschiedene Typen von formalen Systemvarianten einzelner Sprichwort-Komponenten oder des ganzen Sprichworts (z. B. morphologische, grammatische, orthographische Varianten): 
Der Glaube versetzt Berge.

$\rightarrow$ Glaube versetzt Berge.

$\rightarrow$ Glauben versetzt Berge.

$\rightarrow$ Der Glaube kann Berge versetzen.

Die Beschreibungskomponente zur Variation enthält außerdem Angaben zu strukturellen Varianten des ganzen Sprichworts (gemeint sind vor allem syntaktische Umstellungen, Erweiterung und/oder Reduktion des Komponentenbestands, Transformation in eine funktional andere (nichtsatzwertige) Einheit):

Alles hat ein Ende.

$\rightarrow$ Alles hat ein Ende, nur die Wurst hat zwei.

Unter Ersetzung von Komponenten fallen Varianten und Variationsmuster, bei denen eine lexikalische Komponente durch eine oder mehrere andere (möglicherweise musterhaft) ersetzt wird. Diese Austauschbarkeit wird durch die Korpusanalyse ermittelt und muss daher das Kriterium der Verallgemeinerbarkeit erfüllen. Die x-Füller sind daher nur als typische Beispiele anzusehen, es könnten aber auch andere semantisch entsprechende lexikalische Einheiten vorstellbar sein:

Was uns nicht umbringt, macht uns hart.

$\rightarrow$ Was uns (mich/dich...) nicht umbringt, macht uns (mich/dich...) hart.

Der Ton macht die Musik.

$\rightarrow$ Der/die x (Tonfall, Unterton, Zwischentöne) macht/machen die Musik. ${ }^{8}$

Geht mit einer Variante darüber hinaus eine Bedeutungsverschiebung oder -änderung einher, wird dies modellgerecht kommentiert.

\subsubsection{Typische Verwendung im Text}

Diese Angabe vermittelt Beobachtungen $\mathrm{zu}$ typischen textuellen Einbettungen des Sprichworts, die relativ häufig durch die so genannten Sprichwortkonnektoren identifizierbar sind (Ďurčo 2005). Allerdings müssen derartige textuelle Einbettungen mehrfach belegt sein. Die Angaben werden narrativ und möglichst nicht fachspezifisch formuliert. Typische textuelle Einbettungen sind z. B. argumentative Anschlüsse

Der Schein trügt.

$\rightarrow$ Denn/Doch/Aber der Schein trügt.

oder häufige Einfügung von Adverbien, Partikeln usw.

Der Ton macht die Musik.

$\rightarrow$ Der Ton macht halt/eben/freilich die Musik.

oder orthographisch markierter Satzmodus, z. B. Fragesatz oder Imperativ

Ende gut, alles gut.

$\rightarrow$ Ende gut, alles gut?

8 Vgl. http://www.sprichwort-plattform.org/sp/Der\%20Ton\%20macht\%20die\%20Musik\#ref-Der\%20Ton\%20 macht\%20die\%20Musik-8. 


\subsubsection{Volltextbelege}

Die Volltextbelege stammen - wann immer es möglich ist - aus den Korpora einzelner Sprachen und haben die Funktion des lexikographischen Beispiels. Sie illustrieren somit die formellen, grammatischen und semantisch-pragmatischen Eigenschaften des Sprichworts und zeigen auf seine textuelle Verwendung hin. Die Reihenfolge des Belegblocks richtet sich in der Regel nach der Abfolge der lexikographischen Angaben in der Datenbank-Struktur.

\subsubsection{Weitere Belegsuche im Korpus}

Hier werden Suchanfrageformeln registriert, die bei der Datenbank-Erarbeitung für die Suchen nach Sprichwörtern in den jeweiligen Korpora eingesetzt werden. Dadurch wird den Benutzern die Möglichkeit gegeben, das abstrakte Sprichwortmodell, formuliert auf der Basis der formalen korpuseigenen Sprachen, in aktualisierten Korpora neu abzufragen. Außerdem sollen diese Suchanfragen weitere linguistische Parömiologieforschung inspirieren und anregen:

Ausnahmen bestätigen die Regel.

$\rightarrow$ Im Deutschen Referenzkorpus ${ }^{9}$ können über COSMAS II $^{10}$ weitere Belege mit folgender Suchanfrage gefunden werden:

\&Ausnahme /s0 \&bestätigen /s0 \&Regel

Nach denselben Prinzipien wie hier für das Deutsche präsentiert werden äquivalente Sprichwörter in anderen beteiligten Sprachen beschrieben. So besteht die mehrsprachige SprichWort-Datenbank eigentlich aus fünf parallelen, aber untereinander verlinkten einsprachigen Datenbanken.

\section{$4 \quad$ Diskussion}

Fast alle in Kap. 2 beschriebenen Problemstellen einer fachgerechten lexikographischen Behandlung von Sprichwörtern konnten auch bei der Erstellung der SprichWort-Datenbank identifiziert werden. Aus Platzgründen verweise ich an dieser Stelle lediglich auf das Problem der Suche und Selektion von lexikographisch brauchbaren und relevanten Belegen. Postuliert man eine konsequente empirisch gesicherte und korpusbasierte Erhebung sprachlicher Daten als oberstes Prinzip für das Sammeln des lexikographisch relevanten Sprachmaterials, so kommt dem textuellen Beleg die Zentralposition in der lexikographischen Arbeit zu. Er stellt die Basis und den Ausgangspunkt für alle metasprachlichen und metalexikographischen Aussagen dar. Der Beleg verwandelt sich in ein lexikographisch relevantes Beispiel, im Idealfall ein solches, welches die Ausformulierung von verschiedenartigen lexikographischen Angaben unterstützt. Des Weiteren soll es die dem Wörterbuchtyp und -zweck entsprechende Lexik enthalten sowie aktuell und authentisch sein. Leider muss man feststellen, dass die bisherige metalexikographische Forschung noch keine Theorie des lexikographischen Beispiels geliefert hat, wie dies z. B. Wiegand bereits 1977 bemängelte. Bekannt sind seine zwei prinzipiellen Funktionsaspekte: der deskriptive und der normative Aspekt. Der Erstere zeigt sich in der Korrelation mit anderen lexikographischen Angaben im Rahmen eines Wörterbuchartikels, der Letztere in der Festlegung von Kriterien für die Beurteilung seiner Qualität in Bezug auf den Typ und Zweck eines konkreten Wörterbuchs (vgl. Jesenšek 2009, 2009a; Wiegand 1977).

Die fehlenden Qualitätskriterien waren auch bei der Erstellung der SprichWort-Datenbank von Nachteil: Die Lexikographen mussten sich bei der Analyse der Belege und ihrer

\footnotetext{
${ }^{9}$ Siehe http://www.ids-mannheim.de/kl/projekte/korpora/.

10 Siehe http://www.ids-mannheim.de/cosmas $2 /$.
} 
Selektion immer wieder auf die eigene (muttersprachliche) Kompetenz und auf das eigene subjektive Sprachgefühl verlassen. Betrachtet man den Typ und die Zwecke der SprichWortDatenbank (vgl. Kap. 3), so sollten die Belege so ausgewählt werden, dass sie rezeptiv und produktiv orientierte Benutzungsbedürfnisse zugleich decken können. Sie sollten dem Benutzer helfen, die Bedeutung des Sprichworts $\mathrm{zu}$ entschlüsseln, sie illustrieren die Einbettung des Sprichworts in die syntaktische Struktur des Satzes, sie dienen als Nachweis und Vorbild für die aktive Sprichwort-Verwendung in der Fremdsprache. Vor diesem Hintergrund ist bei der Belegselektion besonders darauf $\mathrm{zu}$ achten, dass die enthaltene Lexik dem Sprachniveau der angenommenen Benutzer entspricht (was aufgrund der Authentizität der Belege nicht immer einfach ist) und dass die Belege nicht eine den fremdsprachigen Benutzern ferne und unbekannte Lebensrealität vermitteln, deren entsprechende Semantisierung zusätzliche kulturelle bzw. enzyklopädische Kenntnisse voraussetzen würde. Es hat sich in dieser Hinsicht deutlich gezeigt, dass lediglich das Kriterium der Vorkommenshäufigkeit, welche zugleich die Aktualität eines Sprichworts im gegenwärtigen muttersprachlichen Sprachgebrauch darstellen sollte, nicht genügt, um lexikographisch relevante Belege automatisch liefern zu können, obgleich sie an einem ausgewogenen und somit repräsentativen Sprachkorpus gemessen ist. In puncto Belegselektion sollten die jeweils angenommenen Benutzungssituationen und Benutzungsbedürfnisse der anvisierten Benutzergruppen den absoluten Ausgangspunkt darstellen. Erst auf dieser Basis können detailliertere Selektionskriterien für ein konkretes Wörterbuch oder eine konkrete Datenbank festgelegt werden.

Inwiefern die Auswahl der textuellen Belege in der SprichWort-Datenbank gelungen ist, dies wird in einem weiteren Schritt durch die Erprobung und Evaluation von Seiten einzelner Benutzergruppen ermittelt werden.

\section{$5 \quad$ Fazit und Ausblick}

Da für viele Sprachen moderne, empirisch begründete Sprichwort-Wörterbücher, Datenbanken oder andersartige Ressourcen bisher fehlen, dürfte das kurz vorgestellte Projektvorhaben ein innovatives Konzept zur Erfassung und linguistisch-didaktischen Beschreibung der Sprichwörter mehrerer Sprachen darstellen und zu weiteren ähnlichen Projekten anregen. Das Projekt soll aber auch die Forschung in mehreren Bereichen anregen, vorrangig aber im Bereich der (kontrastiven) linguistischen Sprichwort-Forschung und im Bereich der Metalexikographie.

\section{Literatur}

Baur, Rupprecht S./Chlosta, Christoph (1996): "Sprichwörter. Ein Problem für Fremdsprachelehrer wie -lerner?" Deutsch als Fremdsprache 33: 91-102.

Burger, Harald (2010): Phraseologie. Eine Einführung am Beispiel des Deutschen. 4., neu bearb. Aufl. Berlin: Erich Schmidt Verlag.

Čermák, František (2003): "Paremiological Minimum of Czech: The Corpus Evidence". In: Burger, Harald/Häcki Buhofer, Annelies/Gréciano, Gertrud (eds.): Flut von Texten Vielfalt der Kulturen. Ascona 2001. Zur Methodologie und Kulturspezifik der Phraseologie. Hohengehren, Schneider Verlag: 15-31.

Dobrovol'skij, Dmitrij (2009): "Zur lexikographischen Repräsentation der Phraseme (mit Schwerpunkt auf zweisprachigen Wörterbüchern)". In: Mellado Blanco, Carmen (ed.): Theorie und Praxis der idiomatischen Wörterbücher. Tübingen, Niemeyer: 149-168.

Drosdowski, Günther et al. (eds.) (2001). Duden. Das große Wörterbuch der deutschen Sprache in zehn Bänden. Print- und CD-ROM-Version. Mannheim, Bibliographisches Institut. 
Ďurčo, Peter (2004): "Slovak Proverbial Minimum. The Empirical Evidence". In: Földes, Csaba (ed.): Res humanae proverbiourum et sententiarum. Ad honorem Wolfgangi Mieder. Tübingen, Günter Narr Verlag: 59-70.

Durčo, Peter (2005): Sprichwörter in der Gegenwartssprache. Trnava: Univerzita sv. Cyrila a Metoda v Trnave, Filozofická fakulta.

Grzybek, Peter (1992): "Probleme der Sprichwort-Lexikographie (Parömiographie): Definition, Klassifikation, Selektion". In: Meder, Gregor/Dörner, Andreas (eds.): Worte, Wörter, Wörterbücher. Lexikographische Beiträge zum Essener Linguistischen Kolloquium. Tübingen, Niemeyer: 195-223.

Hallsteinsdóttir, Erla (2006): "Phraseographie". HERMES Journal of Language and Communication Studies 36: 91-128.

Hallsteinsdóttir, Erla (2009): "Zweisprachige Lernerphraseografie aus funktionaler Sicht". In: Mellado Blanco, Carmen (ed.): Theorie und Praxis der idiomatischen Wörterbücher. Tübingen, Niemeyer: 209-231.

Jesenšek, Vida (2005): "Wörterbuch und Übersetzer. Phraseme in der deutsch-slowenischen allgemeinen Lexikographie". Informatologia 38/1-2: 28-35.

Jesenšek, Vida (2006): "Aspekte der Phrasemselektion für didaktische Zwecke. Metodische Überlegungen". Germanistische Linguistik 184/185: 59-71.

Jesenšek, Vida (2007): "Pregovori med preteklostjo in prihodnostjo". In: Jesenšek, Marko (ed.): Besedje slovenskega jezika. Maribor, Slavistično društvo: 276-290.

Jesenšek, Vida (2008): "Phraseologie im zweisprachigen Wörterbuch. Eine Herausforderung für Lexikographen und Übersetzer". Germanistische Linguistik 195/196: 387-404.

Jesenšek, Vida (2009): "Ponazarjalni primer in slovaropisna obravnava frazeologije". Ann, Ser. hist. sociol., 19/: 389-398.

Jesenšek, Vida (2009a): "Phraseologische Wörterbücher auf dem Weg zu Phraseologiedatenbanken". In: Mellado Blanco, Carmen (ed.): Theorie und Praxis der idiomatischen Wörterbücher. Tübingen, Niemeyer: 65-81.

Jesenšek, Vida (2010): "Sprichwörter im Netz - eine Internet-Lernplattform für das Sprachenlernen". In: Mellado Blanco, Carmen (ed.). La fraseografía del S. XXI : nuevas propuestas para el español y alemán. Berlin, Frank \& Timme: 125-148.

Kispál, Tamás (2000): "Einige deutsche Sprichwort-Wörterbücher aus der Sicht des DaFLerners". Jahrbuch der ungarischen Germanistik 1999: 163-181.

Kispál, Tamás (2007): "Sprichwörtersammlungen". In: Burger, Harald et al. (eds.): Phraseologie. Ein internationales Handbuch der zeitgenössischen Forschung. Berlin, New York, de Gruyter: 414-422.

Mieder, Wolfgang (1984): "Geschichte und Probleme der neuhochdeutschen Sprichwörterlexikographie". In: Wiegand, Herbert Ernst (ed.): Studien zur neuhochdeutschen Lexikographie V. Hildesheim, New York, Olms: 307-358.

Mieder, Wolfgang (1989): "Das Sprichwörterbuch". In. Hausmann, Franz Josef et al. (eds.): Wörterbücher. Dictionaries. Dictionaires. Ein internationales Handbuch zur Lexikographie. 1. Teilband. Berlin/New York, de Gruyter: 1033-1044.

Mieder, Wolfgang (1999): "Sprichwörter in den größeren allgemeinen und phraseologischen Wörterbüchern Deutsch-Englisch/Englisch-Deutsch". In: Wiegand, Herbert Ernst (ed.): Studien zur zweisprachigen Lexikographie mit Deutsch IV. Hildesheim, New York, Olms: $1-40$.

Mieder, Wolfgang (2003): "Sprichwörter im GWDS". In: Wiegand, Herbert Ernst (ed.): Untersuchungen zur kommerziellen Lexikographie der deutschen Gegenwartssprache I. Tübingen, Niemeyer: 413-436.

Mieder, Wolfgang (2009): International bibliography of paremiology and phraseology. Berlin etc.: de Gruyter. 
Steyer, Kathrin (2001): "Usuelle Wortverbindungen des Deutschen. Linguistisches Konzept und lexikografische Möglichkeiten". Deutsche Sprache 2/00: 101-125. http://www.idsmannheim.de/pub/laufend/deusprach/abs00-2.html\#1 (Stand: 2.9.2011).

Steyer, Kathrin (2004): "Kookkurrenz. Korpusmethodik, linguistisches Modell, lexikografische Perspektiven". In: Steyer, Kathrin (ed.): Wortverbindungen - mehr oder weniger fest. Berlin/New York, de Gruyter: 87-116. http://www.idsmannheim.de/pub/laufend/jahrbuch/jb2003.html (Stand: 2.9.2011).

Steyer, Kathrin (2009): "Zwischen theoretischer Modellierung und praxisnaher Anwendung. Zur korpusgesteuerten Beschreibung usueller Wortverbindungen". In: Mellado Blanco, Carmen (ed.): Theorie und Praxis der idiomatischen Wörterbücher. Tübingen, Niemeyer: 119-145.

Wiegand, Herbert Ernst (1977): "Nachdenken über Wörterbücher: Aktuelle Probleme". In: Drosdowski, Günther/Henne, Helmut/Wiegand, Herbert Ernst (eds.): Nachdenken über Wörterbücher. Mannheim, Bibliographisches Institut: 51-102. 